\title{
Hour Times Nanomole Per Liter Per Milligram Per Gram
}

National Cancer Institute

\section{Source}

National Cancer Institute. Hour Times Nanomole Per Liter Per Milligram Per Gram. NCI

Thesaurus. Code C117928.

Hour times nanomole per liter, divided by milligram per gram. 